\title{
Mitochondrial functions in five cases of human neuromuscular disorders
}

\author{
A. GIMENO, J. L. TRUEBA, M. BLANCO, AND MARIO GOSAlvez \\ From the Departments of Neurology and of Experimental Biochemistry, \\ Clinica Puerta de Hierro, Facultad de Medicina, Universidad Autónoma, Madrid, Spain
}

SUMMARY We determined the respiration, respiratory control, and Pi:O ratios with different $\stackrel{\mathbb{D}}{\circ}$ substrates in mitochondria isolated from five cases of human neuromuscular disorders (two casesw of central core disease, two cases of neuropathy of Dejerine-Sottas, and one case of Kugelberg- 0 Welander's disease) and compared them with normal human muscle. In all the myopathies studied, $\overrightarrow{\vec{\omega}}$ a severe derangement of the respiratory control with variable derangement of oxidative phosphoryla- $-\infty$ tion was found. This supports the idea that a group of neuromyopathies shares the same biochemicals lesion as the so-called mitochondrial myopathies, forming with them a group of myopathies whicho may be related through a similar biochemical lesion of varying degree. Alternatively, disturbance of mitochondrial functions in a number of myopathies could be considered as a non-specific finding

Despite the frequent occurrence of morphological changes in mitochondria in many types of human neuromuscular disorders (Gruner, 1963; Aleu and Afifi, 1964; Gonatas, Perez, Shy, and Evangelista, 1965; Norris and Panner, 1966; Gonatas, 1967; Engel and Dale, 1968; Chou, 1969), several myopathies have been described in which abnormalities of this organelle were considered to be of major significance (Luft, Ikkos, Palmieri, Ernster, and Afzelius, 1962; Shy, Gonatas, Perez, 1966; Coleman, Nienhuis, Brown, Munsat, and Pearson, 1967; Van Wijngaarden, Bethlem, Meyer, Hülsmann, and Feltkamp, 1967; Price, Gordon, Munsat, and Pearson, 1967; Hulsmann, Bethlem, Meyer, Fleury, and Schellens, 1967; D'Agostino, Ziter, Rallison, and Bray, 1968; Bradley, Hudgson, GardnerMedwin, and Walton, 1969; Spiro, Prineas, and Moore, 1970; Salomon, Esiri, and Ruderman, 1971) and for such cases, the term 'mitochondrial myopathies' has been coined (Price et al., 1967).

Mitochondrial myopathies do not seem to show a uniform pattern of clinical symptoms or microscopic lesions and, therefore, are usually defined by the existence of various kinds of severe alterations of the mitochondrial structure. Five cases of mitochondrial myopathies have been studied biochemically (Luft et al., 1962;
Hülsmann, et al., 1967; Van Wijngaarden et $\stackrel{0}{\text { ब. }}$.) 1967; Spiro et al.,1970). These studies suggesto that mitochondria from mitochondrial myopa thies have a characteristic functional lesion: they are loosely coupled. That is, these mitochondfia lack respiratory control (ratio between respintion in the presence of ADP and respiration in the absence of ADP), while the phosphorylationo (quantity of ADP phosphorylated for each molecule of oxygen consumed) remains unaltered Since mitochondria with good phosphorylatingo ability are frequently referred to as 'wellcoupled', the term 'loosely-coupled' is equi-윽 vocal. It may be preferable to substitute the term 'uncontrolled-well-coupled' (lack of respiratoryo control, well-coupled phosphorylation) for 'loosely-coupled' when defining mitochondrial myopathies.

We have studied the functions of mitochondria? isolated from muscle specimens of five cases of human neuromuscular disorders (none of which was considered as primarily mitochondrial) ing comparison with mitochondria from normals muscle.

\section{METHODS}

The human neuromuscular diseases studied were ภ two cases of central core disease (father and daugh 
ter), two cases of neuropathy of Dejerine-Sottas, and one case of Kugelberg-Welander's disease. The diagnosis of each was arrived at by clinical, histological, histochemical, and electron microscopy investigations. A complete description of the cases will be reported elsewhere. Normal muscle was obtained from three victims of traffic accidents and from normal rats.

Mitochondria were isolated from muscle biopsy specimens of 8 to $15 \mathrm{~g}$, essentially by the method of Chappel and Perry (Ernster and Nordenbrand, 1967) with a variation in the homogenization procedure. The tissue homogenization was as follows: the excised muscle specimen, immersed in $0.15 \mathrm{M} \mathrm{KCl}$, was blotted with filter paper, freed from fat and connective tissue, quickly weighed and immersed in an ice cold isolation medium $(50 \mathrm{mM}$ tris $\mathrm{HCl}$, $100 \mathrm{mM} \mathrm{KCl}, 5 \mathrm{mM} \mathrm{MgCl} 2,1 \mathrm{mM}$ ATP and $1 \mathrm{mM}$ EDTA at pH 7.4). Then the tissue was minced with scissors, washed, and homogenized in a Sorvall omnimixer $(50 \mathrm{ml}$. stainless steel chamber, rotor shaft 17165 , rotor knife 1768 , position 6 of speed control) for 60 seconds. The resulting homogenate was diluted $1: 1$ with isolation medium and further homogenized in a Teflon-glass Elvehjem-Potter homogenizer (clearance $0.15 \mathrm{~mm}$. One to three strokes at 2,000 rpm, depending upon the preparation, to reach a perfect degree of homogenization). The homogenate was diluted with isolation medium and centrifugation of the mitochondria was performed by the method of Chappel and Perry (Ernster and Nordebrand, 1967). The isolated mitochondria were washed at least three times to avoid contamination with myofibrillar ATPase.

Hülsmann, Jong, and Tol (1968) reported that homogenization with a loose-fitting homogenizer releases predominantly subsarcolemmic mitochondria with low respiratory control. Our method of homogenization gives a good yield of high quality mitochondria, as shown by our respiratory control data on normal human muscle (respiratory control from 10 to 16) and on normal rat muscle (respiratory control from 6.7 to 12) (Table 1). The rat data are included for comparison with the data of Hülsmann et al. (1968). The fact that the yield and the respiratory control figures found by us in normal muscle are high implies that, with our method of homogenization, a good fragmentation of the tissue was obtained, at least similar to that achieved by Hülsmann et al. (1968) with tight Teflon homogenizers.

Mitochondrial respiration was determined polarographically in a Clark type oxygen electrode using $0 \cdot 25 \mathrm{M}$ sucrose, $20 \mathrm{mM} \mathrm{KCl}, 7 \mathrm{mM} \mathrm{MgCl}_{2}, 5 \mathrm{mM} \mathrm{Pi}$, and $10 \mathrm{mM}$ tris $\mathrm{HCl}$ as the assay medium $(\mathrm{pH} 7 \cdot 4$, $22^{\circ} \mathrm{C}$ ). The respiratory control and the $\mathrm{Pi}: \mathrm{O}$ ratio were determined as described by Estabrook (1967), by addition of a 200 micromolar aliquot of ADP to mitochondria in state 4 monitored in the oxygen electrode. The figures obtained for each parameter represent the average of three determinations. (Three aliquots of the mitochondrial suspension.) The extreme values did not surpass $7 \%$ of the average in any case.

In all cases of pathological muscle, the $\mathrm{Pi}: \mathrm{O}$ ratios obtained were additionally checked by determination of Pi uptake (Slater, 1967). One aliquot of the mitochondrial suspension was incubated in the oxygen electrode for 40 minutes in the presence of $3 \mathrm{mM}$ ADP. The Pi content at zero and 40 minutes was determined. $\mathrm{Pi}: \mathrm{O}$ ratios determined by $\mathrm{Pi}$ uptake are included in Table 1 between parentheses.

Other scientists working in this field prefer, for the determination of $\mathrm{Pi}: \mathrm{O}$ ratios, the assay of glucose-6-phosphate in a system in which ADP is continuously supplied by ATP, glucose, and hexokinase. Such a system usually yields low respiratory control which indicates the need for a separate determination of respiratory control adding ADP to mitochondria in state 4 . Given the shortage of mitochondria imposed by human muscle biopsies, it is preferable, if respiratory control is detectable, to use a method that simultaneously yields the higher and more accurate figures of respiratory control and $\mathrm{Pi}: \mathrm{O}$ ratio as in the method of Estabrook (1967). This method is able to measure the initial capabilities of mitochondria in a very short time. To illustrate the differences between the three methods of determining $\mathrm{Pi}: \mathrm{O}$ ratios and respiratory control (the ADP:O ratio, Pi uptake and glucose 6 phosphate assay), the data on rat masseter shown in Table 1 were obtained with the three methods. In some preparations of rat muscle, contamination with myofibrillar ATPase cannot be avoided in spite of careful washing. Therefore, it is advisable to supplement the assay medium with EDTA when working with rat muscle. Other supplements, such as albumin or cytochrome $\mathrm{C}$, are, in our opinion, unnecessary. These additions increase the respiration with NADH linked substrates but do not improve respiratory control or $\mathrm{Pi}: \mathrm{O}$ ratios. Comparing the results obtained in rat masseter with the three methods used (Table 1), it can be observed that the respiratory control is lower with the glucose-6-phosphate assay method and that the figure obtained compares well with that obtained by Hülsmann et al. (1968) using the same method.

Mitochondrial protein was determined by the method of Gornall, Bardawill, and David (1969). The concentrations of mitochondrial substrates and other reagents in the assay medium are expressed in the legend to Table 1 . 







\section{RESULTS}

It has been reported that a marked extent of fibrosis and fatty replacement are associated with disturbances of mitochondrial function (Peter, Stempel, and Armstrong, 1970). We observed, in our cases, only slight or discrete fibrosis and fatty replacement of exclusively perimysial localization.

SUMMARY OF HISTOLOGICAL FINDINGS IN EACH CASE Case 1 Central core (father) Quadriceps muscle. There was slight hyperplasia of the perimysial connective tissue with an increase in fatty tissue in some areas, generally perimysial. There was an increase in endomysial fat and connective tissue in only one fascicle. Muscular structure was generally well conserved; the fibres showed diameters of 30 to $80 \mu$ with a few isolated fibres of smaller calibre. There were fibre splitting phenomena, some isolated basophilic fibres, and some isolated signs of nuclear centring. Using histoenzymatic techniques (ATPase), a differentiation in fibre types was seen with type 1 fibres clearly predominating $(75 \%)$. Approximately half of the type 1 fibres showed, with oxidative staining (L.D.H., DPNH, Tr, and SDH), areas lacking oxidative activity; generally single and central, but occasionally eccentric and multiple. Their borders were sharp and surrounded by a halo of greater corpuscular type enzymatic activity. This same lesion appeared with conventional staining, especially in unfixed specimens. With $\mathrm{H}$ and $\mathrm{E}$, homogeneous eosinophilic masses appeared, limited by a slight basophilic halo; with the modified Gomori method (K. Engel), they stained as homogeneous masses surrounded by an increase in the red reticulum. In longitudinal sections, these cores could be seen along the entire muscle fibre. The neuromuscular spindles were normal and did not show cores. The intramuscular nerves were normal. With trichromic staining, many fibres showed perinuclear and subsarcolemmal accumulations of red granulation that stained strongly with oxidative stains. The core structures were confirmed by electron microscopy.

Case 2 Central core (daughter) Quadriceps muscle. There was discrete increase in perimysial connective tissue with very slight endomysial hyperplasia. Scant quantity of adipose tissue was present in the perimysial septa and only isolated deposits endomysially. Muscle fibres of 30 to $80 \mu$ in diameter were found with isolated atrophic fibres of $10 \mu$. Isolated central nuclei were present in some basophilic fibres and there were isolated signs of myofibrillar splitting. Histoenzymatic techniques did not differentiate fibre types; all pertained to type 1 . In the majority of the fibres $(70 \%)$, images of central core similar to the previous cases were seen.

Case 3 Dejerine-Sottasneuropathy-A Quadriceps muscle. Discrete perimysial connective tissue and fatty proliferation without any endomysial changes were found. There was a typical picture of neurogenic atrophy, sometimes fascicular, with groups of fibres $10 \mu$ in diameter. The nonatrophic fibres showed diameters of 30 to $60 \mu$. Nuclei were mostly normal with a few that were vesiculous and showed prominent nucleoli. With PTAH and trichromic methods, perinuclear and subsarcolemmal deposits of granular material that stained heavily for oxidative enzymes were seen. In the subsarcolemmal and perinuclear zones, typical nemalinic rods were frequently observed. They stained intensely red with Masson and intensely blue with PTAH, sometimes forming rows and at other times displaying a random placement. With histoenzymatic techniques, atrophy predominated in type II fibres. With oxidative stains, the majority of the fibres showed positive staining. Abundant target fibres were observed. Electron microscopy demonstrated mitochondria of normal size with crystalline inclusions, increase in intrafibrillar lipid drops (generally in contact with the mitochondria), and myofibrillar fragmentation. A biopsy of the nerve showed a typical 'onion bulb' picture.

Case 4 Dejerine-Sottas neuropathy-B Three muscle biopsies were performed on this patient: in the peroneus longus, quadriceps, and deltoid muscles. They showed a varying degree of neurogenic atrophy without significant hyperplasia of connective or adipose tissue. Perinuclear and subsarcolemmal granular accumulations, strongly positive for oxidative enzymes, were seen. They were less marked, but similar to those described in the previous case. Nemalinic rods were also seen. There was a good differentiation between 
type I and type II fibres. Electron microscopy confirmed the existence of accumulations of zetoide material, myofibrillar fragmentation, and alterations in the number and the morphology of the mitochondria. A biopsy of the sural nerve showed signs of segmental demyelination.

Case 5 Kugelberg-Welander syndrome Quadriceps muscle. There was slight increase in perimysial connective and adipose tissue, without endomysial invasion. A typical picture of neurogenic atrophy was present. There were isolated images of increased muscle fibre volume, with a tendency towards centring of the nuclei. There were subsarcolemmal and perinuclear granular blebs that were occasionally voluminous and stained with trichromic and PTAH stains. They were strongly positive for oxidative stains. With ATPase there was a good differentiation between type I and type II fibres. The atrophy affected both fibre types, but with a predominance of the latter. The intramuscular nerves and spindles were normal.

Electron microscopy showed an increase in the number and volume of the mitochondria, with myofibrillar derangement that started with the A band.

MITOCHONDRIAL FUNCTIONS Table 1 shows the respiration in state 4 (absence of ADP), respiration in state 3 (presence of ADP), the respiratory control, and the $\mathrm{Pi}: \mathrm{O}$ ratios found in the mitochondria of the five pathological cases and in normal muscle with glutamate plus malate or succinate as substrates. This Table also includes the respiration with ascorbate plus $\mathrm{N}, \mathrm{N}, \mathrm{N}^{\prime}, \mathrm{N}^{\prime}$ tetramethyl-P-phenylene diamine (TMPD), the source of the muscle, the yield of mitochondria per gram of muscle, and the age, sex, clinical, and microscopic findings in each case.

The normal muscle showed a respiratory control of 10-16 (glutamate plus malate) or 6.5-9 (succinate) and a $\mathrm{Pi}: \mathrm{O}$ of 2.84-2.94 (glutamate plus malate) or 1.76-1.95 (succinate). These figures approximate to the ideal values for respiratory control and oxidative phosphorylation obtainable with isolated mitochondria. In comparison with these control values, the first case of central core disease (the father) showed a low respiration in state 3 and a relatively high respiration in state 4 with glutamate plus malate or succinate as substrates. Consequently, the res-क्ष piratory control was very low, and since in thiso case the phosphorylation was also very low, almost uncoupled, these mitochondria can be defined as 'uncontrolled-uncoupled' (lack of respiratory control-lack of phosphorylation). The second case of central core disease (the daughter) also showed a marked alteration of. the respiratory control, but the phosphorylation $\overrightarrow{\vec{F}}$ was less affected than in the first case, especiallyo the phosphorylation with glutamate and malateo used as substrates. These mitochondria may be $\frac{\bar{\rho}}{\overline{0}}$ defined as 'uncontrolled-partially coupled'. $\stackrel{\mathbb{1}}{\circ}$ Case A of neuropathy of Dejerine-Sottas showed higher respiration than the other cases; $\vec{\circ}$ moderate derangement of respiratory control and the phosphorylation was slightly affected. Case B $\vec{\omega}$ of Dejerine-Sottas showed a complete lack of respiratory control and a very moderate derange $-\frac{-}{0}$ ment of the $\mathrm{Pi}: \mathrm{O}$ ratios. The fifth case, a. Kugelberg-Welander's disease, showed a veryer low respiratory control (uncontrolled) and phos-\% phorylation was completely conserved. The two last cases, case B of Dejerine-Sottas and the Kugelberg-Welander, present, therefore, a bio-0 chemical pattern completely similar to that designated as 'loosely coupled' in mitochondrial myopathies. (Luft et al., 1962; Hülsmann et al., 196; Van Wijngaarden et al., 1967; Spiro et al. (197\%

There was no significant difference between. the controls and the myopathies in regard to the respiration with ascorbate and TMPD (Table 1)

TABLE 2

SUMMARY OF DATA IN EIGHT MYOPATHIES

\begin{tabular}{|c|c|c|c|}
\hline Diagnosis & $\begin{array}{l}\text { Respiratory } \\
\text { control* }\end{array}$ & $P i: O^{*}$ & Authors \\
\hline $\begin{array}{l}\text { Mitochondrial } \\
\text { myopathy }\end{array}$ & $1 \cdot 25$ & $2 \cdot 6$ & Luft et al. (1962) \\
\hline $\begin{array}{l}\text { Mitochondrial } \\
\text { myopathy }\end{array}$ & $1 \cdot 10$ & $2 \cdot 3$ & Wijngaarden et al. (1967 \\
\hline $\begin{array}{l}\text { Mitochondrial } \\
\text { myopathy }\end{array}$ & $3 \cdot 00$ & $2 \cdot 2$ & Wijngaarden et al. (1967) \\
\hline $\begin{array}{l}\text { Mitochondrial } \\
\text { myopathy } \\
\text { Mitochondrial }\end{array}$ & $1 \cdot 00$ & $1 \cdot 9$ & Hulsman et al. (1967) \\
\hline myopathy & $2 \cdot 30$ & - & Spiro et al. (1970) \\
\hline $\begin{array}{l}\text { Central core } \\
\text { (Case A) }\end{array}$ & $3 \cdot 40$ & $2 \cdot 2$ & Bethlem et al. (1966) \\
\hline $\begin{array}{l}\text { Central core } \\
\text { (Case B) } \\
\text { Myotonic }\end{array}$ & $2 \cdot 30$ & $2 \cdot 9$ & Bethlem et al. (1966) \\
\hline dystrophy & $5 \cdot 00$ & $2 \cdot 3$ & Wijngaarden et al. (1967 \\
\hline
\end{tabular}

* NADH linked substrates. 


\section{DISCUSSION}

For comparison, Table 2 summarizes the data on respiratory control and the $\mathrm{Pi}: \mathrm{O}$ ratios with NADH linked substrates in eight myopathies which have been studied biochemically in the world literature. These are: five mitochondrial myopathies, two central core diseases, and one myotonic dystrophy. The case described by Luft et al. (1962) shows uncontrolled mitochondria with reasonably high phosphorylation. The cases described by Hülsmann et al. (1967) and Van Wijngaarden et al. (1967) show moderate derangement of phosphorylation accompanied by lack of respiratory control, less intense in the second case of Wijngaarden. The case described by Spiro et al. (1970) also shows very low respiratory control. The two cases of central core disease of Bethlem, van Gool, Hülsmann, and Meijer (1966) show low respiratory control and moderately affected or normal phosphorylation. Finally, the case of myotonic dystrophy of Van Wijngaarden et al. (1967) has moderately affected respiratory control and phosphorylation.

Comparing Table 1 and Table 2, it can be concluded that alteration of the respiratory control is common to all myopathies and neuromyopathies studied biochemically so far. This alteration is moderate in our case A of DejerineSottas neuropathy and intense in all cases of central core disease, in cases of mitochondrial myopathy, and in our case B of Dejerine-Sottas neuropathy, as well as in our case of KugelbergWelander's disease. The derangement of phosphorylation, however, did not occur in all these neuromuscular disorders. Our case of KugelbergWelander disease, our case B of Dejerine-Sottas neuropathy, the mitochondrial myopathy reported by Luft et al. (1962), and one central core disease of Bethlem et al. (1966) show practically no alteration of phosphorylation. The rest of the mitochondrial myopathies, one central core disease of Bethlem et al. (1966), our case of central core disease (daughter), and our case A of Dejerine-Sottas neuropathy show moderately affected phosphorylation. Finally, our central core disease (father) shows an almost complete lack of phosphorylation.

As all myopathies studied biochemically have altered respiratory control with variable alteration of oxidative phosphorylation and as our case of Kugelberg-Welander's disease and case B of Dejerine-Sottas neuropathy show a pattern of 'loosely coupled' mitochondria (lack of respiratory control-well conserved phosphorylation) all similar to the most typical mitochondrial myopathy, there is support for the idea that there are neuromyopathies not included in the group of mitochondrial myopathies which show essentially similar biochemical lesions. This could imply that the mitochondrial myopathies along with Kugelberg-Welander's disease, central core disease, neuropathy of Dejerine-Sottas, and possibly myotonic dystrophy, form a group presenting different clinical and histological appearance but related through similar biochemical lesion of varying degree. An alternative possibility could be that the disturbance of mitochondrial function is a relatively non-specific finding seen in a number of muscle diseases. Max (1972) has reported loss of functional activity of mitochondria in experimental diffuse atrophy.

The work reported in this paper was supported by grant CPH 12-080-72 of the Instituto Nacional de Prevision.

\section{REFERENCES}

Aleu, F. P., and Afifi, A. K. (1964). Ultrastructure of muscle in myotonic dystrophy: preliminary observations. American Journal of Pathology, 45, 221-231.

Bethlem, J., Gool, J. van, Hülsmann, W. C., and Meijer A. E. F. H. (1966). Familial non-progressive myopathy, with muscle cramps after exercise. A new disease associated with cores in the muscle fibres. Brain, 89, 569-588.

Bradley, W. G., Hudgson, P., Gardner-Medwin, D., and Walton, J. N. (1969). Myopathy associated with abnormal lipid metabolism in skeletal muscle. Lancet, 1, 495-498.

Chou, S. M. (1969). 'Megaconial' mitochondria observed in a case of chronic polymyositis. Acta Neuropathologia (Berl.), 12, 68-89.

Coleman, R. F., Nienhuis, A. W., Brown, W. J., Munsat, T. L., Pearson, C. M. (1967). New myopathy with mitochondrial enzyme hyperactivity. Journal of the American Medical Association, 199, 624-630.

D’Agostino, A. N., Ziter, F. A., Rallison, M. L., and Bray, P. F. (1968). Familial myopathy with abnormal muscle mitochondria. Archives of Neurology, 18, 388-401.

Engel, A. G., Dale, A. J. D. (1968). Autophagic glycogenosis of late onset with mitochondrial abnormalities. Mayo Clinic Proceedings, 43, 233-279.

Ernster, L., and Nordenbrand, K. (1967). Skeletal muscle mitochondria. In Methods in Enzymology, Vol. 10, pp. 86-94. Academic Press: New York.

Estabrook, R. W. (1967). Mitochondrial respiratory control and the polarographic measurement of ADP:O ratios. In Methods in Enzymology, Vol. 10, pp. 41-47. Academic Press: New York.

Gonatas, N. K. (1967). A generalized disorder of nervous system, skeletal muscle and heart resembling Refsum's 
disease and Hurler's syndrome. 2. Ultrastructure. American Journal of Medicine, 42, 169-178.

Gonatas, N. K., Perez, M. C., Shy, G. M., and Evangelista, I. (1965). Central 'core' disease of skeletal muscle. Ultrastructural and cytochemical observations in two cases. American Journal of Pathology, 47, 503-524.

Gornall, A. G., Bardawill, C. J., and David, M. M. (1949). Determination of serum proteins by means of the biuret reaction. Journal of Biological Chemistry, 177, 751-766.

Gruner, J. E. (1963). Sur quelques anomalies mitochondriales observées au cours d'affections musculaires variées. Comptes Rendus des Séances de la Société de Biologie, 157, 181-182.

Hülsmann, W. C., Bethlem, J., Meijer, A. E. F. H., Fleury, P., and Schellens, J. P. M. (1967). Myopathy with abnormal structure and function of muscle mitochondria. Journal of Neurology, Neurosurgery, and Psychiatry, 30, 519-525.

Hülsmann, W. C., Jong, J. W. de, Tol, A. van (1968). Mitochondria with loosely and tightly coupled oxidative phosphorylation in skeletal muscle. Biochemica et Biophysica Acta, 162, 292-293.

Luft, R., Ikkos, D., Palmieri, G., Ernster, L., and Afzeluis, B. (1962). A case of severe hypermetabolism of nonthyroid origin with a defect in the maintenance of mitochondrial respiratory control. Journal of Clinical Investigation, 41, 1776-1804.

Max, S. R. (1972). Disuse atrophy of skeletal muscle: loss of functional activity of mitochondria. Biochemical and Biophysical Research Communications, 46, 1394-1398.

Norris, F. H., Jr., and Panner, B. J. (1966). Hypothyroid myopathy. Clinical, electromyographical, and ultrastructural observations. Archives of Neurology, 14, 574-589.
Peter, J. B., Stempel, K., and Armstrong, J. (1970). Bio- Z chemistry and electron microscopy of mitochondria in muscular and neuromuscular diseases. In Muscle Diseases. Proceedings of an International Congress, Milan, 1969, pp. 228-235. Edited by J. N. Walton, N. Canal, and G. Scarlato. International Congress Series No. 199. Excerpta ${ }_{\infty}$ Medica: Amsterdam.

Price, H. M., Gordon, G. B., Munsat, T. L., and Pearson, C. M. (1967). Myopathy with atypical mitochondria in type I skeletal muscle fibres. Journal of Neuropathology. and Experimental Neurology, 26, 475-497.

Shy, G. M., Gonatas, N. K., and Perez, M. (1966). Two childhood myopathies with abnormal mitochondria. 1. Megaconial myopathy. 2. Pleoconial myopathy. Brain, 89, 흠 133-158.

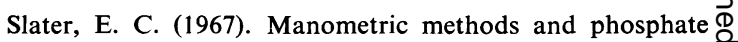
determination. In Methods in Enzymology, Vol. 10, pp. 19- נר 29. Academic Press: London.

Salomon, M. A., Esiri, M. M., and Ruderman, N. B. (1971). $\overrightarrow{0}$ Myopathic disorder associated with mitochondrial ab- normalities, hyperglycaemia, and hyperketonaemia. Lancet, $\vec{\omega}$ 2, 290-293.

Spiro, A. J., Prineas, J. W., and Moore, C. L. (1970). A new mitochondrial myopathy in a patient with salt craving. Archives of Neurology (Chicago), 22, 259-269.

Wijngaarden, G. K. van, Bethlem, J., Meijer, A. E. F. H Hülsmann, W. C., and Feltkamp, C. A. (1967). Skele్ㅣㅇㅇㅇ muscle disease with abnormal mitochondria. Brain, 糖, o 577-592. 